\title{
Preserving generalist skills in the NHS - is a medical hospitalist service the way forward? The Canadian experience and how it could translate to the NHS
}

\author{
Authors: Lynn Lambert and Peter Jamieson
}

\begin{abstract}
Aims
A description of a successful hospitalist service in a publically insured health system and its applicability to the UK health service with a view to better management of hospital capacity and rural staff shortfall.
\end{abstract}

\section{Methods}

Review of medically qualified hospitalists in Calgary, Canada.

\section{Results}

Hospitalists in Canada are general physicians whose main focus of practice is the inpatient care of medically unwell patients in teaching or community-based hospitals, some of whom have a community-based office practice in addition. The hospitalist is the 'most responsible physician' for the admitted patient and does not work under supervision of another consultant. Most of these physicians have their specialist certification in family medicine (general practice) but their main focus is the care of the acutely admitted patient in the non-critical care setting.

The first hospitalist service in Canada was at Calgary's Peter Lougheed Hospital in 1998 and there are now over 100 programmes across Canada. The Canadian Society of Hospital Medicine (CHSM) was founded in 2001 to promote best practice and in 2015 the CSHM published the Core Competencies in Hospital Medicine document which has much in common with the core competencies for UK trainees in general medicine.

The Foothills Hospital in Calgary is the main teaching hospital in Calgary and has a team of 32 hospitalists who are trained in family medicine and look after around 200 inpatients and 70-80 subacute patients. They cover the majority of general medical inpatient diagnoses, elderly care patients and some palliative care and work on a rota to cover both day and night. There is close collaboration with the specialist services, both medical and surgical, on a consultation basis, with the hospitalist retaining the role of the most responsible physician. Very complex patients can be transferred to specialist services which co-exist with the hospitalist service. General internal medicine physicians work on the medical teaching unit and take the very complicated patients.

\section{Conclusion}

The hospitalist service, as seen in Calgary, has much to be commended to the NHS as a way of providing doctor-based, generalist services for the bulk of hospital medical admissions which are currently overwhelming NHS facilities. In the NHS, entry to the service could be via general practice training or after core medical training and Membership of the Royal Colleges of Physicians without the need for every generalist to be a fully trained specialist in another specialty. 